\title{
Functional Predictions of Long Noncoding RNAs by MiRNAs in Populus Euphratica Oliv.
}

\author{
Wei-wei YAN ${ }^{1}$, Hao ZOU ${ }^{1}$, Sheng-jie LUO ${ }^{1}$, Zhen-zhong ZHOU ${ }^{1}$, \\ Shao-wei QIN ${ }^{1, a, *}$ and Li-feng ZHAO ${ }^{1,2, b}$ \\ ${ }^{1}$ College of Life Sciences, Tarim University, Alar 843300, People's Republic of China \\ ${ }^{2}$ Key Laboratory of Protection and Utilization of Biological Resources in Tarim Basin, \\ Tarim University, Alar 843300, People's Republic of China
}

\author{
aqinshaowei2005@126.com \\ blifengz2011@126.com \\ ${ }^{*}$ Corresponding author
}

Keywords: IncRNA, Function, Populus euphratica Oliv., miRNA, ceRNA.

\begin{abstract}
Long noncoding RNAs (lncRNAs) are refer as noncoding transcript RNAs and longer than 200 nucleotides (nt) in length but shorter than 10,000 nt. LncRNAs are involved in a variety of biological processes, such as development, stress response. However, the functions of lncRNAs in plants are largely unclear. MicroRNAs (miRNAs) can regulate gene expression at transcriptional or post-transcriptional level in eukaryotes by sequence complementation. At present, the research on noncoding RNAs targeted by miRNAs is seldom explored in plants. Populus euphratica Oliv. ( $P$. euphratica) is one of ideal model plants for studying development and stress resistance. To uncover the potential functions of lncRNAs in $P$. euphratica, the tender leaves of $P$. euphratica were analyzed using sequencing, and the data including lncRNAs and miRNAs were obtained, followed by bioinformatics methods to predict the potential binding sites of 258 mature miRNAs at $4460 \mathrm{lncRNAs}$ in $P$. euphratica. It was found that 159 lncRNAs can act as the targets of 33 miRNAs. The corrections of miRNAs-mRNAs-lncRNAs were constructed and the functions of lncRNAs were predicted according to the roles of targets (mRNAs) of corresponding miRNAs. Our results indicated that lncRNAs of $P$. euphratica can regulate development, metabolism, and stress response and regulation process by decoying corresponding miRNAs.
\end{abstract}

\section{Introduction}

Long noncoding RNAs (lncRNAs) are long transcripts of more than 200 nucleotide (nt) but less than 10,000 nt that lack a coding sequence (CDS) [1, 2, 3]. LncRNAs can regulate gene expression at transcriptional and post-transcriptional levels and involving in the regulation of reproductive process, organ morphogenesis and stimulus response et al [4-8]. At present, the studies about lncRNAs major focus on animal, lesser on plant.

The poplar species Populus euphratica Oliv. (P. euphratica) grows almost exclusively in the desert, is the only tree species in the Taklimakan Desert (China) [9]. $P$. euphratica has a high tolerance for salinity, drought, cold, and wind, meanwhile also exhibits a typical heterophylly $[9,10]$. All above characters of $P$. euphratica make it one of ideal model plants for study development and stress resistance. The types and functions of lncRNAs in P. euphratica are unknown still. In this study, to explore the functions of lncRNAs acting as miRNA targets or decoys, the noncoding RNA (ncRNA) 
sequencing with $P$. euphratica leaves were performed at first, then, a genome-scale analysis among miRNAs and lncRNAs was performed. The functions of lncRNAs were predicted and annotated according to the ceRNA hypothesis at last.

\section{Materials and Methods}

\section{Plant Materials and NcRNA Sequencing}

The tender leaves, including linear, lanceolate, oval and broad-oval leaves, were collected from $P$. euphratica in Alar, Xinjiang province, China. These samples were disposed immediately according to the methods of Zhao and Qin [10]. Total RNAs were extracted using the mirVana miRNA Isolation Kit (Ambion) following the manufacturer's protocol. RNA integrity was evaluated using the Agilent 2100 Bioanalyzer (Agilent Technologies, Santa Clara, CA, USA). The samples with RNA Integrity Number (RIN) $\geq 7$ were subjected to the subsequent analysis. Strand-Specific RNA libraries were constructed using TruSeq Stranded Total RNA LT - (with Ribo-Zero Plant) according to the manufacturer's instructions. Then these libraries were sequenced on the Illumina sequencing platform (HiSeqTM 2500 or other platform) and $150 \mathrm{bp} / 125 \mathrm{bp}$ paired-end reads were generated. Small RNA libraries and Sequencing were following the procedure described by Zhao and Qin [10].

The transcripts with splicing of each sample were combined and screened as lncRNAs with Cuffmerge, and then compared to the $P$. euphratica genome (ftp://ftp. ncbi.nlm.nih.gov/genomes/all/GCF_000495115.1_PopEup_1.0/) with Cuffcompare Software. For miRNAs, clean reads were screened the length of 21-22 nt as miRNAs, and located to refer sequence with bowtie. Combined with miREvo Softwareand mirdeep2 Software [11], the functions of novel miRNAs were analyzed. All sequencing programs were performed by OE Biotech (China, Shanghai).

\section{Predictions of MiRNA Targets and the Corrections between LncRNAs and MRNAs}

The miRNA targets of IncRNAs or mRNAs were predicted using miRanda Software, the IncRNAs or mRNAs were selected when the value of score $\geq 140$. Then the corrections between lncRNAs and mRNAs were constructed basing on their interaction with miRNAs.

\section{Functional Predictions and Annotation of LncRNAs}

The sequences of all of the listed mRNAs connected with lncRNAs by miRNAs were compared to the transcriptome of Arabidopsis thaliana (GCF_000001735.3 _TAIR10_ rna.fna.gz, https://www.ncbi.nlm.nih.gov/genome/?term=rabidopsis+thaliana), then the homologous genes of these mRNAs in Arabidopsis thaliana were obtained, at last the gene symbols of these homologous genes were submitted to GO analysis. The overrepresented GO terms in the "biological process" category was obtained using Fisher's exact test and the Bonferroni multiples test $(P$-value $<0.05)[11]$.

\section{Results}

\section{The Expression of LncRNAs and MiRNAs in P. euphratica}

The tender samples of linear, lanceolate, oval and broad-oval leaves were analyzed using a sequencing technique at germination stage. It was found that 4460 lncRNAs 
(including 442 novel lncRNAs and 4018 annotated lncRNAs) and 258 miRNAs (including 91 novel miRNAs and 167 annotated miRNAs) were expressed in $P$. euphratica (Fig. 1).
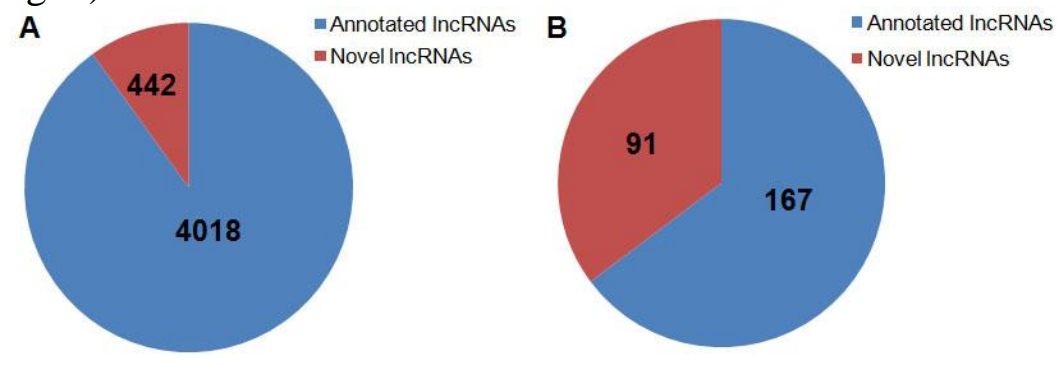

Fig. 1 The number of lncRNAs (A) and miRNAs (B) which expressed in P. euphratica.

\section{The Corrections between MiRNAs and LncRNAs in P. euphratica}

All of 4460 lncRNAs and 258 miRNAs of $P$. euphratica were analyzed with miRanda Software. It was found that 159 lncRNAs can act as the targets of 33 miRNAs. To more easily describe the corrections between miRNAs and lncRNAs, these 33 miRNAs were named with symbol numbers (SNs) (Table 1).

Table 1. The symbol numbers of miRNAs in this paper

\begin{tabular}{|l|c|l|l|l|c|}
\hline \multicolumn{1}{|c|}{ miRNAs } & SN & \multicolumn{1}{|c|}{ miRNAs } & \multicolumn{1}{c|}{ SN } & \multicolumn{1}{|c|}{ miRNAs } & SN \\
\hline NW_011499846.1_1010 & 1 & ptc-miR156a & 12 & ptc-miR396f & 23 \\
\hline NW_011499846.1_779 & 2 & ptc-miR156g & 13 & ptc-miR398a & 24 \\
\hline NW_011499850.1_2817 & 3 & ptc-miR160e-3p & 14 & ptc-miR403c-5p & 25 \\
\hline NW_011499851.1_3625 & 4 & ptc-miR160e-5p & 15 & ptc-miR408-3p & 26 \\
\hline NW_011499858.1_5634 & 5 & ptc-miR162a & 16 & ptc-miR473a-3p & 27 \\
\hline NW_011499986.1_17983 & 6 & ptc-miR169n-3p & 17 & ptc-miR477a-3p & 28 \\
\hline NW_011500067.1_22368 & 7 & ptc-miR3627b & 18 & ptc-miR477a-5p & 29 \\
\hline NW_011500122.1_25017 & 8 & ptc-miR394a-5p & 19 & ptc-miR6424 & 30 \\
\hline NW_011500297.1_28413 & 9 & ptc-miR395a & 20 & ptc-miR6427-3p & 31 \\
\hline NW_011500297.1_28413* & 10 & ptc-miR395b & 21 & ptc-miR6427-5p & 32 \\
\hline NW_011500300.1_28478 & 11 & ptc-miR396c & 22 & ptc-miR6462c-5p & 33 \\
\hline
\end{tabular}

The corrections between lncRNAs and miRNAs were displayed in Table 2. It was showed that some lncRNAs can only act as targets of one miRNA, such as TCONS_36417, it is only the target of NW_011500297.1_28413, but some lncRNAs were targets of multiple miRNAs, such as XR_843263.1, it is the targets of 18 miRNAs (Table 2).

\section{The Functions of LncRNAs Predicted by MiRNAs}

According to the GO, the targets (mRNAs) of one miRNA involved in dozens to hundreds of biologic processes, it is difficult to determine what the major function of a miRNA is. So that, for a miRNA, the ratio of target counts which involved in a biologic process with the counts of all Arabidopsis thaliana genes which involved in this biologic process was obtained, then the top 5 biologic processes were used to character this miRNA. The top GO terms of these 33 miRNAs involved in add up to 89; these 89-biologic processes can be categorized as development, metabolism, stress response and regulation. The types of these 33 miRNAs involved in were displayed in Fig. 2. Additionally, the top 1 biologic process of miRNAs which involved in was list in Table 3.

Combined Table 2 with Fig. 2, it was showed that 127 lncRNAs participate in jointly the biologic process regulation of $P$. euphratica by decoying miRNA 6,8 , 


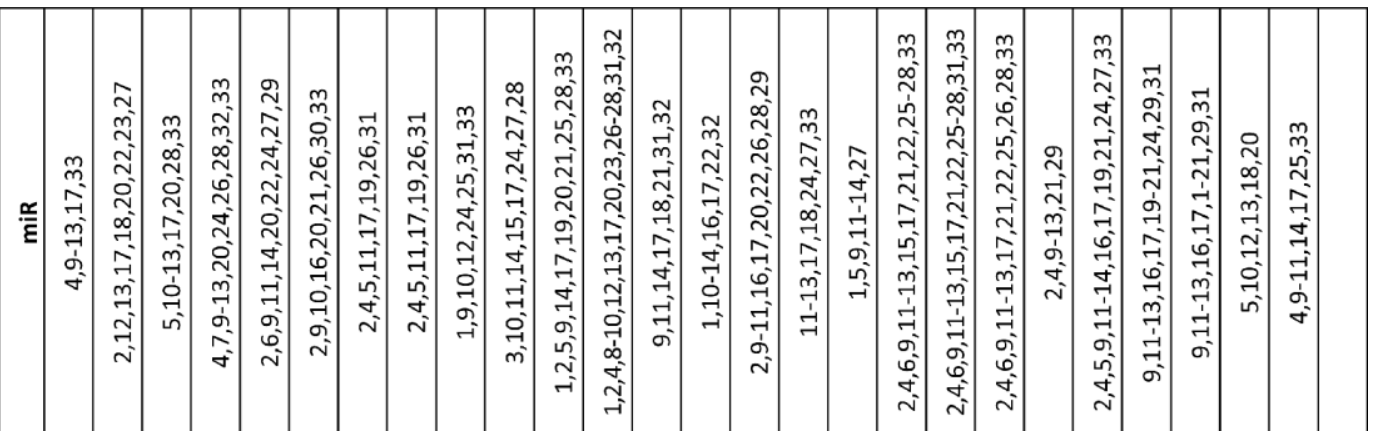

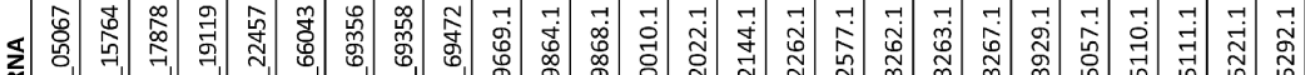

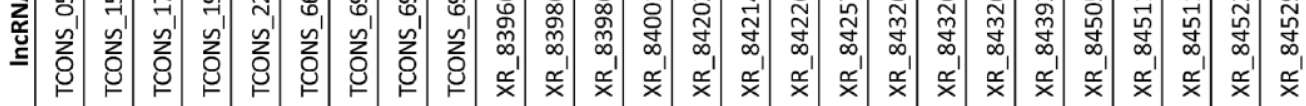

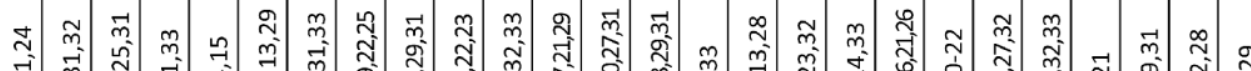

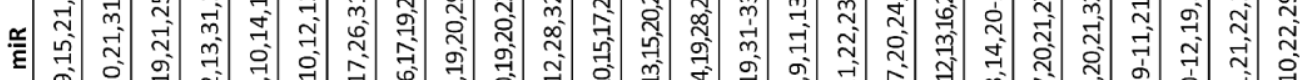

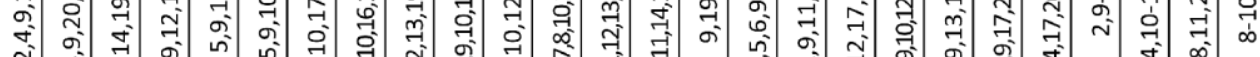

4 象

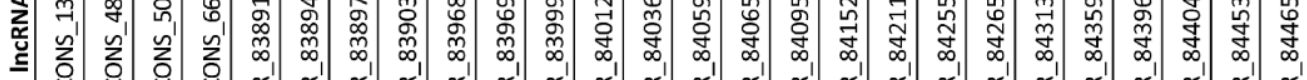

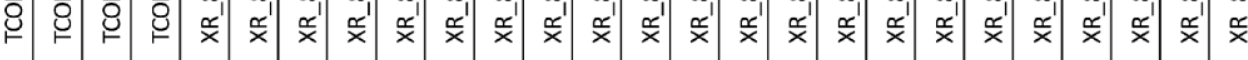

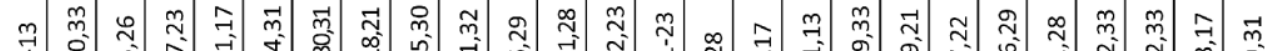

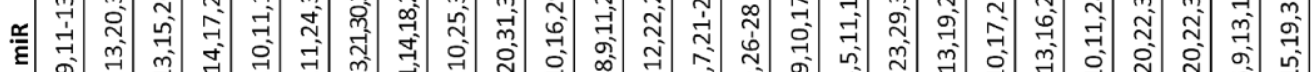
跑

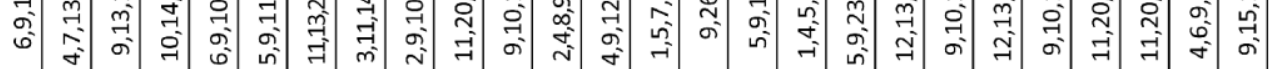

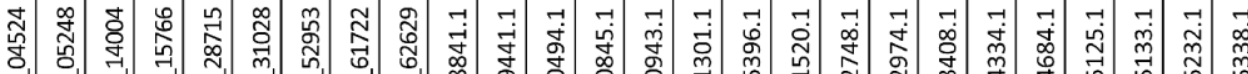

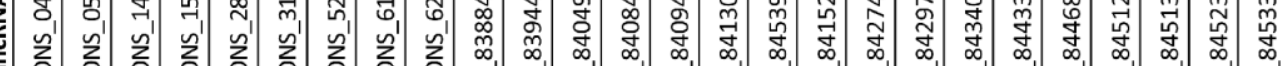

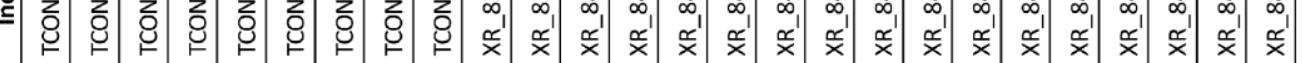

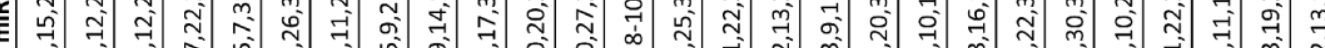

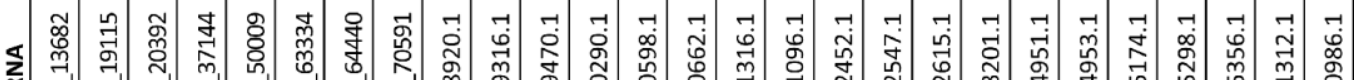

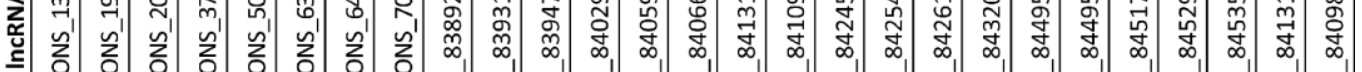

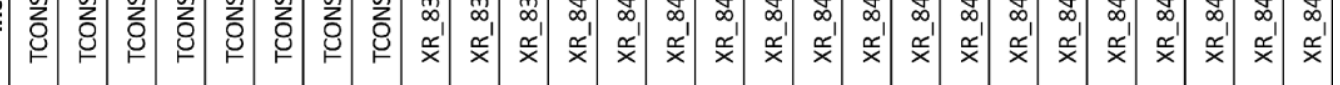

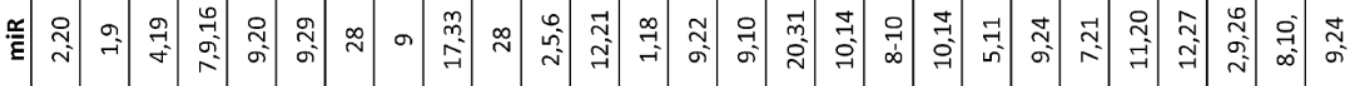

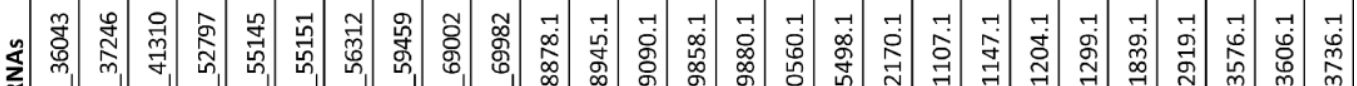

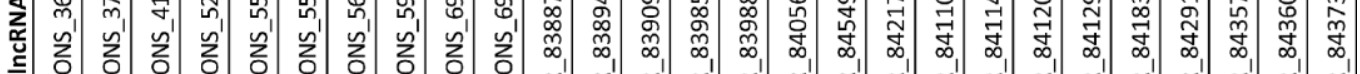

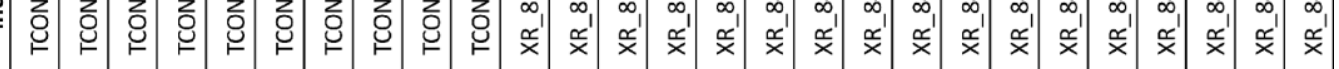

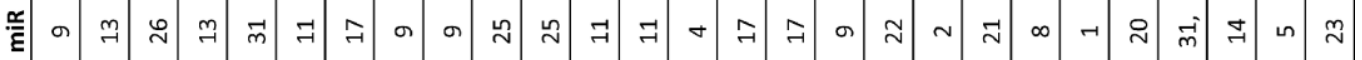

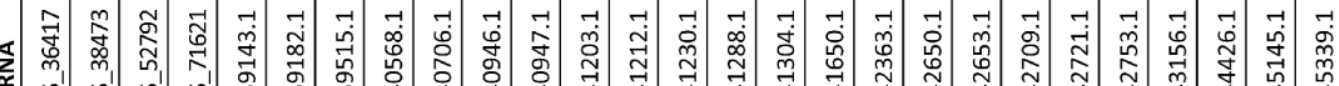

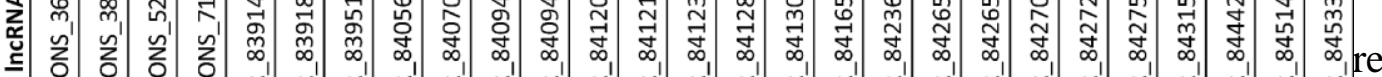

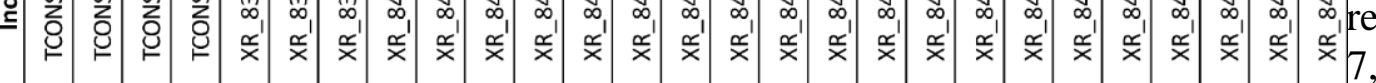


19, 22, 26, 28, 29 and 33; 109 lncRNAs can decoy miRNA 1, 3, 7, 10, 12, 15, 16, 25, 27, 28, 30 and 32, and participate in jointly the stress response of $P$. euphratica; 151 IncRNAs can decoy miRNA 1, 3-10, 12-17, 19-24, 26, 27, 29 and 31-33 respectively, and play a role in $P$. euphratica metabolism together.

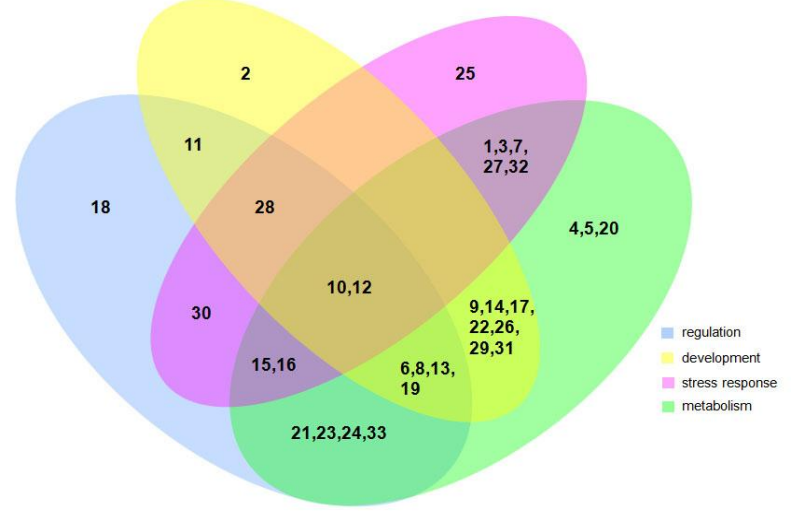

Fig. 2. The types of 33 miRNAs involved in biologic processes of P. euphratica. The different colors show the different types of biological processes. The symbol of a miRNA in a color showed the biologic process which participating in only belongs to this type. If a miRNA in the overlap areas of multiple colors, it is indicated that this miRNA can participate in multiple biological processes simultaneously.

Table 3. The top 1 biologic process of miRNAs which involved in

\begin{tabular}{|c|c|c|c|c|c|c|c|c|c|c|c|}
\hline miR & $\begin{array}{c}\text { GO } \\
\text { accession }\end{array}$ & Ratio & $\begin{array}{c}\boldsymbol{P} \\
\text { value }\end{array}$ & miR & $\begin{array}{c}\text { GO } \\
\text { accession }\end{array}$ & Ratio & $\begin{array}{c}\boldsymbol{P} \\
\text { value }\end{array}$ & miR & $\begin{array}{c}\text { GO } \\
\text { accession }\end{array}$ & Ratio & $\begin{array}{c}\boldsymbol{P} \\
\text { value }\end{array}$ \\
\hline 1 & 0009809 & 0.10 & 0.0373 & 12 & 0060429 & 0.28 & 0.0465 & 23 & 0006066 & 0.10 & 0.0382 \\
\hline 2 & 0030048 & 0.32 & 0.0031 & 13 & 0048532 & 0.17 & 0.0057 & 24 & 0051093 & 0.10 & 0.0030 \\
\hline 3 & 0019438 & 0.01 & 0.0002 & 14 & 0060429 & 0.28 & 0.0193 & 25 & 1901615 & 0.05 & 0.0278 \\
\hline 4 & 0098660 & 0.08 & 0.0008 & 15 & 0046274 & 0.24 & 0.0142 & 26 & 0048440 & 0.11 & 0.0419 \\
\hline 5 & 0006816 & 0.21 & 0.0090 & 16 & 0010105 & 0.25 & 0.0325 & 27 & 0009808 & 0.12 & 0.0061 \\
\hline 6 & 0006928 & 0.15 & 0.0182 & 17 & 0070588 & 0.32 & 0.0089 & 28 & 0044242 & 0.13 & 0.0118 \\
\hline 7 & 0006955 & 0.03 & 0.0220 & 18 & 0009890 & 0.04 & 0.0092 & 29 & 0009913 & 0.33 & 0.0171 \\
\hline 8 & 0009790 & 0.03 & 0.0067 & 19 & 0009658 & 0.11 & 0.0032 & 30 & 0010646 & 0.05 & 0.0076 \\
\hline 9 & 0060429 & 0.44 & 0.0042 & 20 & 0009687 & 0.27 & 0.0212 & 31 & 0048646 & 0.09 & 0.0037 \\
\hline 10 & 0030048 & 0.32 & 0.0147 & 21 & 0070588 & 0.32 & 0.0040 & 32 & 0071395 & 0.10 & 0.0243 \\
\hline 11 & 0030048 & 0.37 & 0.0006 & 22 & 0030048 & 0.32 & 0.0043 & 33 & 0034220 & 0.08 & 0.0010 \\
\hline
\end{tabular}

Note: Ratio means the ratio of target counts which involved in a biologic process with the counts of all Arabidopsis thaliana genes which involved in this biologic process. $P$ value means the $P$ value of corresponding GO term.

\section{Discussion}

With their importance in regulating gene expression, lncRNAs have garnered significant attention in life science field [3]. Although increasing lncRNAs have been predicted and identified in plants [3], the amounts and functions of lncRNAs in $P$. euphratica are unknown until today. In this study, 4460 lncRNAs of $P$. euphratica were obtained by sequencing technique, and then the functions of 159 lncRNAs were predicted by ceRNA hypothesis.

According to the GO enrichment with the targets of miRNAs and the major lncRNAs involved in metabolism (Fig. 2), it was showed that metabolism not only provided materials for life, but also was a basis of many biologic processes. For 
example, Kalve et al. found that the cytoplasmic growth is mainly based on macromolecular synthesis and therefore consumes amount of energy, so that metabolism can affect cell growth [12]. P. euphratica has typical heterophylly [9, 10], 140 lncRNAs involved in development process, this result indicated that these lncRNAs maybe play a key role in the P. euphratica leaf development. 109 lncRNAs involved in stress response, it was indicated that these lncRNAs involved in regulation resistance of salt, temperature and drought stresses, enable $P$. euphratica to live in the desert [10]. 127 lncRNAs involved in regulation process, illustrating that lncRNAs can also affect the expression of numerous genes by decoying limited miRNAs.

\section{Conclusions}

In this study, 4460 lncRNAs of $P$. euphratica were obtained by sequencing technique, and then the functions of $159 \operatorname{lncRNAs}$ were predicted by ceRNA hypothesis. It be suggested that 151 lncRNAs could regulate metabolic process by decoying 27 miRNAs respectively; 149 lncRNAs could regulate development by decoying 16 miRNAs respectively; 127 IncRNAs were involved in regulation process by decoying 16 miRNAs respectively; 109 lncRNAs were involved in stress response by decoying 12 miRNAs respectively. All above results indicated that lncRNAs of $P$. euphratica play vital roles in metabolism, leaf development, stress response et al., and enable $P$. euphratica to live in desert.

\section{Acknowledgement}

This research was financially supported by the National Natural Science Foundation of China (31660298) and Training Plan to Innovation and Enterprise of National University Students (201610757034).

\section{References}

[1] J. Ponjavic, C.P. Ponting, G. Lunter, J. Genome Res. 17(2007) 556-565.

[2] C.P. Ponting, P.L. Oliver, W. Reik, J. Cell. 136(2009) 629-641.

[3] C. Fan, Z. Hao, J. Yan, G. Li, BMC Genomics J. 16 (2015) 1-19.

[4] T.R. Mercer, M.E. Dinger, J.S Mattick, J. Nat Rev Genet. 10(2009) 155-159.

[5] S.Y. Ng, R. Johnson, L.W. Stanton, J. EMBO. 31(2012) 522-533.

[6] P.G. Hawkins, K.V. Morris, J. Transcription. 1(2010) 165-175.

[7] Q.H. Zhu, M.B. Wang, J. Genes (Basel). 3(2012) 176-190.

[8] J.L. Rinn, H.Y. Chang, J. Annu Rev Biochem. 81(2012) 145-166.

[9] J. Si, T. Zhou, W. Bo, F. Xu, R. Wu, J. BMC Genet. 15(2014) 1-11.

[10] L.F. Zhao, S.W. Qin, J. Plant Growth Regul. 81(2017) 231-242.

[11] J. Zhou, Q. Xiong, H. Chen, C. Yang, Y. Fan, J. Front Mol Neurosci. 10(2017) 1-22.

[12] S. Kalve, D. De Vos, G.T. Beemster, J. Front Plant Sci. 5(2014) 1-25. 